\title{
Los cambios de la tecnología usada en educación a través del tiempo
}

\author{
Changes in the technology used in education over time
}

Mudanças na tecnologia usada na educação ao longo do tempo

\section{María Dolores Villamar Irrazabal}

maria.villamari@ug.edu.ec

https://orcid.org/0000-0002-6762-5019

Universidad de Guayaquil, Guayaquil-Ecuador

\section{Omar Efrén Otero Agreda \\ omar.oteroa@ug.edu.ec \\ https://orcid.org/0000-0003-0822-6509}

Universidad de Guayaquil, Guayaquil-Ecuador

\author{
María Alejandrina Nivela Cornejo \\ maria.nivelac@ug.edu.ec \\ https://orcid.org/0000-0002-0356-7243 \\ Universidad de Guayaquil, Guayaquil-Ecuador
}

Artículo recibido 15 de julio 2021, arbitrado y aceptado 18 de agosto 2021 y publicado 13 de diciembre 2021

\section{RESUMEN}

Esta investigación tuvo por objetivo describir los cambios ocurridos en la tecnología usada en educación desde el año 1980 hasta la actualidad en Ecuador. Metodológicamente se realizó un estudio documental, tipo informativo, se realizó el estudio bibliométrico logrando obtener 57 documentos luego de la aplicación de criterios de selección. Como unidad de análisis se usó el tema, realizando un análisis de contenido temático. Como resultado se describieron las categorías: software educativo, soporte multimedia, Videojuegos, Internet, Tecnología inalámbrica, Learning Management System, Realidad virtual, y, Sistemas de gestión de contenidos y redes sociales. Se pudo deducir de la bibliografía estudiada que las tecnologías usadas en educación en Ecuador no van a la par de las tecnologías emergentes. Se concluyó que estos cambios han transitado desde las tecnologías más elementales hasta las más complejas y actualizadas; sin embargo, su incorporación a la educación siempre es lenta en comparación con la evolución tecnológica.

Palabras clave: Educación; Internet; Learning Management System; Tecnología; Tecnologías emergentes

\section{ABSTRACT}

The objective of this research was to describe the changes that occurred in the technology used in education from 1980 to the present in Ecuador. Methodologically, an informative documentary study was carried out, the bibliometric study was carried out, obtaining 57 documents after applying selection criteria. The theme was used as the unit of analysis, carrying out a thematic content analysis. As a result, the categories were described: educational software, Multimedia support, Videogames, Internet, Wireless technology, Learning Management System, Virtual reality, and, Content management systems and social networks. It could be deduced from the bibliography studied that the technologies used in education in Ecuador do not go hand in hand with emerging technologies. It was concluded that these changes have gone from the most elementary technologies to the most complex and updated; however, their incorporation into education is always slow compared to technological evolution.

Key words: Education; Internet; Learning Management System; Technology; Emerging technologies

\section{RESUMO}

O objetivo desta pesquisa foi descrever as mudançasocorridasnatecnologiautilizada na educação de 1980 até a atualidade no Equador. Metodologicamente, realizouse um estudo documental informativo, foi realizado o estudo bibliométrico, obtendo-se 57 documentos após aplicação de critérios de seleção. O tema foi utilizado como unidade de análise, realizando uma análise de conteúdo temática. Como resultado, foram descritas as categorias: software educacional, suporte multimídia, Videogames, Internet, Tecnologia sem fio, Sistema de gerenciamento de aprendizagem, Realidade virtual e, Sistemas de gerenciamento de conteúdo e redes sociais. Pode-se deduzir da bibliografia estudada que as tecnologias utilizadas na educação no Equador não andam de mãos dadas com as tecnologias emergentes. Concluiu-se que essas mudanças passaram das tecnologias mais elementares às mais complexas e atualizadas; no entanto, sua incorporação na educação é sempre lenta em comparação com a evolução tecnológica

Palavras-chave: Educação; Internet; Sistema de gestão de aprendizagem; Tecnologia; Tecnologias emergentes 


\section{INTRODUCCIÓN}

A nivel mundial la educación ha pasado por transformaciones propias de la realidad histórica, cultural, política, social y económica de cada país; sin embargo, existe un factor común, y es la incorporación de la tecnología, en distintas formas y épocas, la cual ha evolucionado y en consecuencia generado cambios en los paradigmas educativos; donde el factor resistencia siempre ha estado presente.

Pensar en educación, las formas de aprender o de enseñar, sin tener en cuenta la tecnología, la computación, las TIC, y la Web 2.0 es prácticamente inexistente (Adell, 2010). De esta manera cada espacio temporal en educación tiene asociada una o varias tecnologías relacionadas a su época, y que influyen en las posibilidades, limitaciones, procesos y condiciones de la enseñanza y el aprendizaje en las cuales pretenden impactar (Castañeda, Salinas y Adell, 2020).

En esta investigación el tema abordado se relaciona con esos cambios o evolución experimentada por la tecnología usada en la educación, para ello se describen algunos antecedentes, donde se especifica la problemática general estudiada.

Para el año 1994, Domínguez (1994), realizó una investigación titulada: Perspectivas del desarrollo de la tecnología educativa hacia el año 2000, donde cuyo objetivo consistió en vislumbrar las posibilidades y limitaciones del uso de las tecnologías en la educación de Iberoamérica hacia el año 2000; en este analizó el uso de la televisión y de la computación como apoyo al sector educativo; en este trabajo destaca que los materiales más usados eran los textos y medios impresos, los cassette de audios y el retroproyector, las diapositivas y los diaporamas.
Ya finalizando la década de los noventa del siglo XX, Marqués (1997), presenta un trabajo titulado "La informática en la enseñanza primaria, donde hace notar la importancia y la utilidad de esta en la educación", describiendo sus finalidades básicas. Entre sus conclusiones destaca que la informática puede proporcionar programas para agilizar la gestión de institutos educativos, y puede ser aprovechada como recursos para docentes y estudiantes ya que facilita determinados procesos educativos, todo dependiendo de la metodología de uso. Según su perspectiva "un buen programa no basta" (p. 17).

A inicio del siglo XXI, Sánchez (2000) presenta una investigación titulada: Aprender con Internet: Mitos y Realidades, en la cual habla del Internet como recurso pedagógico para el aprendizaje, destacando entre sus consideraciones: la tecnología e Internet no van a modificar los paradigmas educativos, seguramente podrán ampliar o profundizar costumbres. "Todo depende del antiguo dilema, la tecnología como una clara solución a un problema educativo o la tecnología como una solución esperando por un problema educativo" (p. 14).

Por su parte, Cabero (2001) en su libro “Tecnología educativa, diseño y utilización de medios en la enseñanza" presenta una evolución de esta descrita a través de cinco momentos. El primero, constituye los tiempos iniciales o prehistoria; el segundo, referido a la incorporación de los medios audiovisuales y de comunicación de masas; el tercero, marcado por la introducción de la psicología conductista y el trabajo con la tecnología instruccional; el cuarto momento, signado por la introducción del enfoque sistémico a la educación; y el último, tiene nuevas orientaciones vinculadas a la psicología cognitiva. 
Area (2001a) presenta un trabajo sobre las redes de ordenadores en la enseñanza universitaria: hacia los campus virtuales, donde analizó en qué medida las redes y la Internet modifican los fines, métodos y procesos de enseñanza desarrollados en la universidad. También describió las formas de utilización e integración pedagógica de las redes de computadores en la docencia universitaria tanto presencial como a distancia on line o virtual; finaliza explicando el concepto y características de un "campus virtual".

Para contextualizar, mientras en algunos países como Estados Unidos, México y Chile, ya se estaba trabajando con fuerza el tema de la incorporación de la tecnología reciente en la educación en todos los niveles, en Ecuador, la primera iniciativa oficial que propuso incorporar las tecnologías de la información y comunicación en el sistema educativo comenzó en el 2002 cuando se implementó un programa de capacitación para docentes en el uso pedagógico del computador. Ya para el año 2011 las escuelas públicas se estaban dotando a de equipamiento de aulas de informática con acceso a internet y con miras al modelo tecnológico $1 \mathrm{x} 1$. (Peñaherrera, 2011).

En la investigación presentada por Aguirre y Ruiz (2012), se hace referencia a la educación a distancia y virtual, para la cual desde su comienzo:

Se han empleado recursos tecnológicos para proveer experiencias de formación asistidas por plataformas virtuales que contribuyan a la formación de individuos, especialmente a través de los llamados Learning Managment System (lms), entre los que se pueden destacar: Moodle, Claroline, Dokeos, Blackboard, entre otros, que ofrecen recursos de comunicación como foros, chats, wikis, mensajería instantánea, además de herramientas para administrar la actividad del estudiante, como talleres, tareas, y centro de calificaciones, entre otros. (Aguirre y Ruiz, 2012, p. 123).

Espinel (2020) refiere en su investigación la existencia de distintas herramientas tecnológicas para docentes y estudiantes, tales como: a) las destinadas al acceso a la información: laptop, IPad, Smartphone, teléfonos inteligentes; b) las de conectividad y coordinación de tareas: bluetooth, internet, intranet, Wi-Fi; c) las expositivas: Prezi, Power Point, Youtube y d) las plataformas tecnológicas: Moodle, buscadores como google académico, google libros, entre otros.

Además, se cuenta con entornos virtuales de formación tales como: WebCT, Learning Space, Edustance; herramientas de autor, como: Hot Potatoes, Quia!, Clic; y software tales como: Perception y Quiz Factory (sic). Sin embargo, con todas las ventajas del uso educativo de las herramientas tecnológicas, tienen una restricción vinculada a la facilidad de acceso y conectividad. (Espinel, 2020).

Por su parte, Area (2001b) indica que en la mayoría de las aulas de los sistemas escolares a nivel mundial se enseña con una tecnología propia del siglo XV, siendo nula la utilización de recursos, artefactos, estrategias y lenguajes informáticos del siglo XX, y esta historia se repite aún en el 2021 en muchos países. De igual forma, Gregorini (2017) en su investigación reportó que las instituciones educativas que habían sido dotadas de netbooks tenían dificultades para el uso de internet debido a la imposibilidad de proveer de WiFi al salón de clases. 
De esta revisión teórica se nota que dentro de la tecnología usada en educación durante los últimos años se destacan entre los más relevantes el software educativo, los soporte multimedia, videojuegos, Internet, Tecnología inalámbrica, Learning Management System, Realidad virtual, y Sistemas de gestión de contenidos y redes sociales. En este sentido surgen las interrogantes: ¿las tecnologías usadas en educación van a la par de las tecnologías emergentes?; ¿cuáles han sido los cambios ocurridos en la tecnología usada en educación desde el año 1980 hasta la actualidad en Ecuador? Esta situación conllevó a realizar la presente investigación, la cual tuvo por objetivo: describir los cambios ocurridos en la tecnología usada en educación desde el año 1980 hasta la actualidad en Ecuador.

Esta investigación se justifica por el hecho de que prácticamente a diario surgen nuevas tecnologías que pueden ser usadas de forma valiosa en educación para generar aprendizajes más efectivos, tal como lo plantea Carrillo (2014), los docentes como creadores de diseños instruccionales deben ser competentes y mantenerse actualizados sobre la existencia, uso y manejo de recursos tecnológicos proporcionados por las TIC y sus tecnologías emergentes, con la finalidad de innovar en la aplicación de estrategias que permitan alcanzar los objetivos de aprendizaje.

De esta forma el propósito de este artículo consiste en aportar evidencias científicas sobre si las tecnologías usadas en educación van a la par de las tecnologías emergentes; y sobre los cambios ocurridos en la tecnología que se ha usado en educación en las últimas cuatro décadas. Por tanto puede considerarse una contribución al cuerpo teórico epistémico de la informática educativa como disciplina emergente.

\section{MÉTODO}

Para lograr el objetivo propuesto se realizó una investigación documental la cual según Arias (2006) consiste en un proceso de búsqueda, recuperación, análisis, crítica, e interpretación de datos secundarios; es decir, información documentada por otros investigadores.

Fue informativa, este tipo de investigación documental pretende informar sobre una temática de relevante relacionada con un tema específico, la cual para este trabajo fueron los cambios ocurridos en la tecnología usada en educación. Se describe el objeto de estudio con todos los detalles posibles, ordenando y seleccionando toda la información existente (Montagud, 2020).

La búsqueda bibliográfica quedó conformada por el universo de fuentes documentales asociadas a las tecnologías usadas en educación desde 1980 hasta 2021. Para garantizar la fiabilidad y validez de las fuentes seleccionadas sólo se consideraron libros y artículos publicados en revista científicas reconocidas y Tesis, de acuerdo con la información obtenida en Internet y su búsqueda mediante Google Académico, al respecto se encontraron 243.000 resultados.

Se establecieron criterios de selección de las fuentes encontradas en la búsqueda inicial, los cuales permitieron refinar la búsqueda, estos fueron los siguientes:

- Utilización del intervalo específico de tiempo: 1980-2021.

- Considerar las investigaciones a texto completo.

- Relacionados con el uso de tecnologías en educación. 
Al analizar todo el universo poblacional encontrado se realizó el estudio bibliométrico, logrando como resultado un censo poblacional de 57 documentos, con los cuales se desarrolló el estudio correspondiente.

La unidad de análisis considerada fue el tema; estableciendo el siguiente: uso de la tecnología en educación. Se escogieron categorías de contenido como objeto de análisis, para ello se procedió a realizar una primera lectura tanto de los resultados de investigaciones, así como también de los artículos escogidos. Posteriormente se identificó y se separaron las categorías presentadas en la matriz de la Tabla 1.

Tabla 1. Matriz relacional entre unidades de análisis y categorías.

\begin{tabular}{lll}
\hline Unidad de análisis & Categorías & Fuente \\
\hline
\end{tabular}

$$
\text { Software educativo }
$$

Soporte multimedia

Videojuegos

Internet

Uso de la

tecnología en

educación

Tecnología inalámbrica

Learning Management System

Realidad virtual

Sistemas de gestión de contenidos y redes sociales
Arriaga, Pescador y Portaencasa (1993); Marqués (1996); Alvarado y Oña (1998); Cataldi, Lage, Pessacq y García (1999); Gros, (2000); Benites, Herrera, Salas, y Cuenca (2009); Vidal, Gómez y Ruiz (2010); Macías-Mendoza, (2015); Nivela, Agreda, Izquierdo y Carrera (2018); Mex et al. (2021)

Campos et al. (1994); Campaña (2012); Chedraui (2020); Espinoza et al. (2020); Gallo (2020)

Crespo, Greenberg y Busselle (1996); Marcos (1997); Lazo y Gabelas (2008); Mantilla, Rodríguez y Villacís (2020); Santo y Chávez (2020); Ocampo-Pazos, et al. (2020); Tontaquimba (2021)

Ramírez (2006); López-Jiménez (2010); Gil, Rosero y Raza (2011); Botello-Peñaloza (2015); Chamba-Eras, Coronel-Romero, y Labanda-Jaramillo (2016); Mendoza-Zambrano, Tirado-Morueta y Marín-Gutiérrez (2017); Espín-Álvarez y Freire-Muñoz (2019); Cazarez (2021).

Rogel y Verduga (2013); Marizaca (2015); Alvarado-Salinas y Núñez-Izaguirre (2017); Lagos et al. (2019); Tubay y Oviedo (2020); Cobos, Simbaña y Jaramillo (2020).

Gallego, Cela y Hinojosa (2011); Caicedo et al. (2015); Guanoluisa y Herrera (2018); Peláez-López et al. (2018); García, Pinargote y García (2019); Cueva y Zarate (2020); Ocampo-Alvarado y Ullauri-Ugarte (2021)

Villalba y Durán (2010); Urquiza et al. (2016); Samaniego (2016); Magallanes et al. (2021)

Area (2008); Castañeda (2009); Tapia-León, Peñaherrera-Larenas y Cedillo-Fajardo (2015); Leyva et al. (2018); Hernández (2018); Humanante-Ramos, Fernandez-Acevedo y Jimenez (2019); Hermann-Acosta, Apolo y Molano-Camargo (2019); Sangucho y Baque (2021); Martínez-Sanz, y Arribas-Urrutia (2021); AlcívarMoreira y Yánez-Rodríguez (2021). 
Seguidamente se realizó una búsqueda según las categorías establecidas para su posterior lectura, análisis e interpretación.

Se utilizó el análisis de contenido temático, el cual "considera la presencia de términos o conceptos con independencia de las relaciones surgidas entre ellos" (Arbeláez y Onrubia, 2014: 20). El proceso para este análisis se realizó de forma deductiva; es decir, buscando en el documento estudiado las categorías establecidas y construidas mediante referentes teóricos. Díaz (2018). El instrumento de recolección de la información usad fue una matriz de análisis del contenido.

\section{DESARROLLO Y DISCUSIÓN}

Luego de la revisión de la bibliografía seleccionada y del establecimiento de las categorías, la Figura 1 muestra un mapa mental de las tecnologías más utilizadas en educación desde 1980 hasta la actualidad.

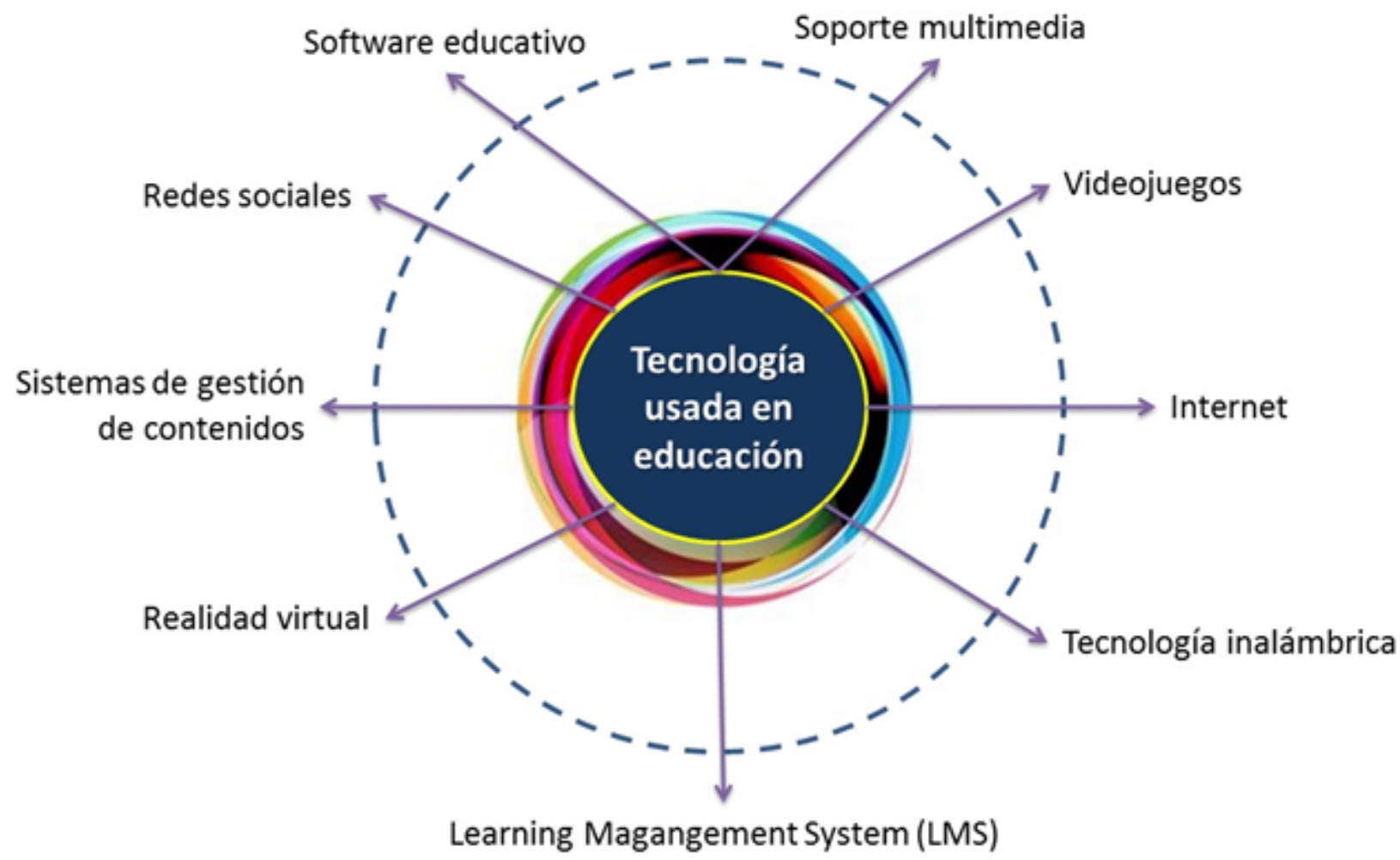

Figura 1. Tecnología más usada en educación desde 1980.

Tal como se evidencia en la Figura 1, la tecnología usada en educación ha experimentado cambios que están relacionados con las TIC que han surgido en cada época, sin embargo, en la literatura estudiada se nota que estas variaciones no siempre van a la par.
En el caso del uso del software educativo, Ecuador reporta investigaciones como las de Alvarado y Oña (1998); Benites, Herrera, Salas, y Cuenca (2009); Macías-Mendoza, (2015); Nivela, Agreda, Izquierdo y Carrera (2018) donde se evidencian resultados de su aplicación 
en educación en distintos niveles del sistema educativo; sin embargo, el trabajo más antiguo data de 1998, cuando según García (2002), el origen de la introducción y utilización de estos en educación es de la década de los sesenta, caracterizados por una secuencia lineal de presentación de pantallas y admitiendo una interacción limitada con los usuarios.

Con relación al uso de Soporte multimedia, estos son definidos como productos basados en sistemas multimedia; es decir, que ofrecen combinaciones y tratamientos múltiples: de texto, imágenes y sonido, en diferentes medios de comunicación, así como hardware y software que tengan relación con estos (Chedraui, 2020). Al respecto, se encontraron los trabajos de Campos et al. (1994) en España; y en Ecuador: Campaña (2012); Chedraui (2020); Espinoza et al. (2020) y Gallo (2020); así se observan en el Ecuador investigaciones de reciente data (siglo XXI), teniendo que según Pérez (1994) la incorporación de medios audiovisuales a las aulas es considerada una significativa contribución de las TIC a la educación. Mientras en España ya en 1994, según Matas (2001), algunos editores se estaban encargando de crear espacios multimedia para mantener cautivo su mercado lector; y se realizaban debates académicos sobre las transformaciones de las bibliotecas y del sistema educativo.

Los Videojuegos fueron otra categoría estudiada, para ello se tienen las investigaciones de: Crespo, Greenberg y Busselle (1996); Marcos (1997); Lazo y Gabelas (2008); Mantilla, Rodríguez y Villacís (2020); Santo y Chávez (2020); OcampoPazos, et al. (2020); Tontaquimba (2021), teniendo para Ecuador investigaciones del año 1996 donde se reportó que los jóvenes y niños ecuatorianos usaban videojuegos; sin embargo, no era con fines educativos sino distractivos, las relacionadas con el uso en educación son del siglo XXI, de data relativamente reciente; aunque su uso se remonta a los años 80 del siglo XX.

Internet, estudiado a través de los trabajos de: Ramírez (2006); López-Jiménez (2010); Gil, Rosero y Raza (2011); Botello-Peñaloza (2015); ChambaEras, Coronel-Romero, y Labanda-Jaramillo (2016); Mendoza-Zambrano, Tirado-Morueta y MarínGutiérrez (2017); Espín-Álvarez y Freire-Muñoz (2019); Cazarez (2021), tal como puede verse en estas investigaciones el uso de internet en la educación ecuatoriana toma auge en este siglo; aun cuando Valencia, Milla y Machado (1995) afirman que el uso de internet en la educación remonta a inicios de los años 90 cuando la Fundación Nacional de Ciencia (NSF: National Science Foundation), una agencia del gobierno norteamericano, "promueve el acceso educacional universal, mediante la creación de conexiones, en los campus, pero solamente si éstos tienen un plan para la extensión del acceso" (p. 70). Sin embargo, en Ecuador, en varios estudios realizados acerca del uso del internet se observó que fue a partir del 2015 cuando su uso aumentó, llegando a alcanzar un 50,5\% a nivel nacional. (Peláez-López et al., 2018)

Con la llegada de internet y su uso masificado en toda la sociedad, surgen la Tecnología inalámbrica, estudiadas mediante los trabajos de: Rogel y Verduga (2013); Marizaca (2015); Alvarado-Salinas y Núñez-Izaguirre (2017); Lagos et al. (2019); Tubay y Oviedo (2020); Cobos, Simbaña y Jaramillo (2020). Estas son utilizadas a nivel mundial y han permitido la apertura de las telecomunicaciones, entre ellas están: "WiFi, Bluetooth, ZigBee y Z-Wave" (Adeva, 2021). 
Como puede observarse, en Ecuador, toma auge el uso de esta tecnología en educación ya en el siglo XXI, con todo y que esta data de 1971 cuando un grupo de investigadores de la Universidad de Hawaii, coordinados por Norman Abramson crearon el primer sistema de conmutación de paquetes mediante una red de comunicación por radio, llamada ALOHA, esta es la primera red de área local inalámbrica WLAN, formada por 7 computadoras. (Historia de la Informática, 2010).

El Learning Management System (LMS), fue estudiado a través de las investigaciones de Gallego, Cela e Hinojosa (2011); Caicedo et al. (2015); Guanoluisa y Herrera (2018); Peláez-López et al. (2018); García, Pinargote y García (2019); Cueva y Zarate (2020); Ocampo-Alvarado y Ullauri-Ugarte (2021); como se puede notar, su incorporación al área educativa data de este siglo XXI, aun cuando su origen remonta a 1995, convirtiéndose en significativos porque hacen que las instituciones educativas ofrezcan estos servicios a los estudiantes y profesores (García-Peñalvo y Seoane, 2015).

La Realidad virtual, estudiada mediante los trabajos presentados por: Villalba y Durán (2010); Urquiza et al. (2016); Samaniego (2016); Magallanes et al. (2021); tal como se evidencia, su incorporación al área educativa data de la segunda década del siglo XXI; sin embargo, su origen está enmarcado según la literatura a finales de la década de 1950, cuando Morton Heilig (1926-1997) diseñó la primera experiencia multisensorial virtual, desarrollando un equipo denominado Sensorama, el cual combinaba video, audio, vibración, viento e incluso olores. (Olguin, Rivera y Hernández, 2006).

Los Sistemas de gestión de contenidos y redes sociales, fueron estudiados a través de las investigaciones de: Area (2008); Castañeda (2009); Tapia-León, Peñaherrera-Larenas y Cedillo-Fajardo
(2015); Leyva et al. (2018); Hernández (2018); Humanante-Ramos, Fernández-Acevedo y Jimenez (2019); Hermann-Acosta, A., Apolo, D., y MolanoCamargo, M. (2019); Sangucho, D., y Baque, E. (2021); Martínez-Sanz, R., y Arribas-Urrutia, A. (2021); Alcívar-Moreira, Á., y Yánez-Rodríguez, M. (2021). Con los cuales se evidencia que en Ecuador su incorporación a la educación es de fecha reciente, en este siglo XXI.

Sin embargo, las redes sociales aunque parecen un fenómeno naciente, surgieron antes que la Web 2.0. Se puede decir que su fecha de nacimiento data de 1997, cuando se inaugura la web SixDegrees. com. Posteriormente aparecieron webs como Ryze. com en 2001, Tribe.net en 2003 y LinkedIn en 2003. Con la explosión de la Web 2.0, llega Youtube y Facebook en 2004. (Ros-Martín, 2009).

\section{CONCLUSIÓN}

En esta investigación se describieron los cambios ocurridos en la tecnología usada en educación desde el año 1980 hasta la actualidad en Ecuador. Además, tal como se pudo notar en la bibliografía estudiada, las tecnologías usadas en educación en Ecuador no van a la par de las tecnologías emergentes.

Estos cambios han transitado desde las tecnologías más elementales hasta las más complejas y actualizadas; sin embargo, su incorporación a la educación siempre es lenta en comparación con la evolución tecnológica; esto puede ser atribuido a la falta de disposición a su uso como herramienta de impacto positivo para el proceso de enseñanza aprendizaje, o de preparación de los docentes, aunque también pueden intervenir otros factores que merecen ser investigados, por tanto se recomienda continuar develando estos elementos intervinientes. 


\section{REFERENCIAS}

Adell, J. (2010). Educación 2.0. En C. Barba y S. Capella (Eds.), Ordenadores en las aulas. La clave es la metodología. Graó

Aguirre, G.y Ruiz, M.(2012). Competencias digitales y docencia: una experiencia desde la práctica universitaria. Innovación educativa (México, DF), 12(59), 121-141. http://www.scielo.org. $\mathrm{mx} /$ scielo.php?script=sci_arttextypid=S166526732012000200009ylng=esytlng=es

Alcívar-Moreira, Á., y Yánez-Rodríguez, M. (2021). Las redes sociales y su influencia en el rendimiento académico de los estudiantes de educación básica media. Polo del Conocimiento, 6(4), 40-53. https://www.polodelconocimiento. com/ojs/index.php/es/article/view/2537/5293

Alvarado, E. y Oña, G. (1998). Estudio de técnicas multimedia y aplicación de sus elementos eficientemente en el desarrollo de software educativo multimedia (Bachelor's thesis). Pontificia Universidad Católica del Ecuador, Sede Ambato. http://repositorio.pucesa.edu.ec/ handle/123456789/332

Alvarado-Salinas, Y. y Núñez-Izaguirre, P. (2017). Educación en seguridad criptográfica para redes inalámbricas con tecnologías $\mathrm{WiFi}$, Bluetooth y WIMAX (original). Olimpia: Publicación científica de la facultad de cultura física de la Universidad de Granma, 14(43), 231-241. https://dialnet.unirioja.es/servlet/ articulo? codigo $=6210806$

Arbeláez, M., y Onrubia, J. (2014). Análisis bibliométrico y de contenido. Dos metodologías complementarias para el análisis de la revista colombiana Educación y cultura. Revista de Investigaciones UCM, 14(23), 14 - 31

Area, M. (2001a). Las redes de ordenadores en la enseñanza universitaria: hacia los campus virtuales. En A. García-Valcárcel (coord.): Didáctica Universitaria. La Muralla, 231-260. https://www.researchgate.net/profile/ManuelArea/publication/242448948_Capitulo_7 LAS_REDES_DE_ORDENADORES_EN_ LA_ENSENANZA_UNIVERSITARIA_ HACIA_LOS_CAMPUS_VIRTUALES/
links/56f95f5708ae38d710a2febe/Capitulo-7LAS-REDES-DE-ORDENADORES-EN-LAENSENANZA-UNIVERSITARIA-HACIALOS-CAMPUS-VIRTUALES.pdf

Area, M. (2001b). Usos y prácticas con medios y materiales en el contexto escolar. De la cultura impresa a la cultura digital. Kikiriki. Cooperación educativa, (61), 39-44. http://www. quadernsdigitals.net/datos/hemeroteca/r_42/ nr_477/a_6370/6370.pdf

Area, M. (2008). Las redes sociales en internet como espacios para la formación del profesorado. Razón y Palabra, (63). https://www.redalyc.org/ articulo.oa?id=199520798005

Arias, F. (2006). El Proyecto de Investigación. Introducción a la metodología científica. $6^{\mathrm{a}}$ Edición. Episteme

Arriaga, J., Pescador, F., y Portaencasa, R. (1993). Guía de software para la enseñanza de la electrónica. RED. Revista de educación y formación profesional a distancia. Revista Nuevas tecnologías, 101-108. https:// redined.educacion.gob.es/xmlui/bitstream/ handle/11162/80486/00820093002105. pdf? sequence $=1$ yisAllowed $=y$

Adeva, R. (14 de abril de 2021). Tecnologías inalámbricas: diferencias y usos de $\mathrm{WiFi}$, Bluetooth, Zigbee y Z-Wave. Adslzone. https:// www.adslzone.net/reportajes/tecnologia/ estandares-conexion-inalambrica/

Benites, G., Herrera, A., Salas, C., y Cuenca, J. (2009). Representación de las Funciones Trigonométricas en el Software Educativo como es el Programa Geogebra. Revista de Informática Educativa y Medios Audiovisuales, 6(12), 1317. http://laboratorios.fi.uba.ar/lie/Revista/ Articulos/060612/A4jul2009.pdf

Botello-Peñaloza, H. (2015). Determinantes del acceso al internet: Evidencia de los hogares del Ecuador. Entramado 11(2), 12-19, http://dx.doi. org/10.18041/entramado.2015v11n2.22205

Cabero, J. (2001). Tecnología educativa. Diseño y utilización de medios en la enseñanza. Paidós 
Caicedo, C., Rodríguez, A., Caicedo, F., Acuña, R., y Delgado, G. (2015). Plataforma Virtual a través de una infraestructura basada en el Sistema de gestión de aprendizajes. Revista Científica Sinapsis, 2(7). https://doi.org/10.37117/s. v2i7.67

Campos, B., Lozano, J., Hueros, A., Graván, P. y Cembellín, R. (1994). La formación vía satélite ante las demandas de la formación ocupacional. Pixel-Bit. Revista de medios y educación, 3, 29-45. https://www.researchgate. net/profile/Ana-Hueros/publication/28103169_ La_formacion_via_satelite_ante_las_ demandas_de_la_formacion_ocupacional/ links / $58527 \mathrm{~d} 4508$ aef7 d 030 a 4 e $9 \mathrm{~d} 6 / \mathrm{La}$ formacion-via-satelite-ante-las-demandas-dela-formacion-ocupacional.pdf

Campaña, M. (2012). Diseño de un plan de comunicación con sistemas multimedia interactivos en entornos de e-learning síncrono para la estructura organizacional y sistema de educación a distancia de la Universidad Internacional del Ecuador, de Quito (Doctoral thesis), Universidad Internacional del Ecuador, Quito, Ecuador. https://repositorio.uide.edu.ec/ handle/37000/2051

Carrillo, D. (2014). Competencias TIC de los docentes para la enseñanza mediante entornos virtuales en educación superior. El caso de la Universidad de los Andes-Venezuela: evaluación y diseño de un plan de formación (Doctoral thesis), Universitat Rovira I Virgili, Tarragona. https://www.tdx.cat/handle/10803/285330

Castañeda, L. (2009). El cibersalón: Educación superior y YouTube. Chasqui, 106, 76-81. http:// hdl.handle.net/10469/13792

Castañeda, l.; Salinas, J. y Adell, J. (2020). Hacia una visión contemporánea de la Tecnología Educativa. Digital Education Review, 37, 240268. http://greav.ub.edu/der/

Cataldi, Z., Lage, F., Pessacq, R., y García, R. (1999). Ingeniería de software educativo. In Proceedings del $\mathrm{V}$ Congreso Internacional de Ingeniería Informática (pp. 185-199). https://d1wqtxts1xzle7. cloudfront.net/44903590/ c-icie99- ingenierias oftwareeducativo-with-coverpage-v2.pdf Expires $=1628543493 y$ Signature $=$ J F y V u s a J 2 P X f S q Z x G d Y K I u 4 s 8 f 6 Th 49 X zh p 6 S 4 y l ma g Nks OdczHiMhjA-DghFHOllQ 1 U 5 y 7 E Ttjg 9 Y D eXh C KB Rwg s xfVko P7E6rJeBPu4n96UoslxHXECwEjpfGSGEo y i I Q E L G G W L x 80 Z F c 8 o z t S jYBBY5vVFMxWsusrvxRuY-VM7e6h57SDo4CwEN-x11fSq6cNSwIEuaQZBPP9ifPV jKL3Z82YAxhO04nKAsGqc Fd6CiYwL6j45 vVM5Qxd5JWXbfs WjM4fWj0F7674kqf3EX a M $7 \mathrm{E} \sim \mathrm{K}$ o t P N a J 4 c 7 q R Z 6 f S 8 F T 0 yOoeam7QpnM3m0 SaNTSUo2jIE3KMLjQ_ yKey-Pair-Id=APKAJLOHF5GGSLRBV4ZA

Cazarez, J. (2021). Competencias del profesorado universitario en relación al uso de tecnologías de la información y comunicación: un estudio de caso en la Universidad Central del Ecuador (Tesis Doctoral). Universidad de Alicante, España. http://hdl.handle.net/10045/115056

Chamba-Eras, L., Coronel-Romero, E., y LabandaJaramillo, M. (2016). Usabilidad Web: situación actual de los portales Web de las Universidades de Ecuador. Sexta Conferencia de Directores de Tecnología de Información, TICAL 2016 Gestión de las TICs para la Investigación y la Colaboración, Buenos Aires, 13 al 15 de septiembre de 2016. http://dspace. redclara.net:8080/bitstream/10786/1105/1/ Usabilidad\%20Web\%20situaci\%C3\%B3n\%20 actual\%20de\%20los\%20portales $\% 20 \mathrm{Web} \% 20$ de $\% 201$ as $\% 20$ Universidades $\% 20$ de $\% 20$ Ecuador.pdf

Chedraui, L. (2020). La multimedia en la formación profesional en la educación superior en el Ecuador. Res Non Verba Revista Científica, 10(1), 1-14. https://doi.org/10.21855/ resnonverba.v10i1.317

Crespo, M., Greenberg, B. y Busselle, R. (1996). Los jóvenes y su orientación hacia los medios de comunicación en Brasil, Chile y Ecuador. CIC. Cuadernos de Información y Comunicación, (2), 107-123. https://revistas. ucm.es/index.php/CIYC/article/download/ CIYC9696110107A/7448 
Cobos, J., Simbaña, V., y Jaramillo, L. (2020). El mobile learning mediado con metodología PACIE para saberes constructivistas. Sophia, colección de Filosofía de la Educación, (28), 139164. https://doi.org/10.17163/soph.n28.2020.05

Cueva, T. y Zarate, V. (2020). El uso de un sistema de gestión de aprendizaje en el modelo educativo medio del Ecuador. Polo del Conocimiento, 5(8), 1421-1459. DOI: 10.23857/pc.v5i8.1749 https://polodelconocimiento.com/ojs/index. $\mathrm{php} / \mathrm{es} /$ article/view/1749

Díaz, C. (2018). Investigación cualitativa y análisis de contenido temático. Orientación intelectual de revista Universum. Revista General de Información y Documentación, 28(1), 119-142. DOI: https://doi.org/10.5209/RGID.60813

Domínguez, M. (1994). Perspectivas del desarrollo de la tecnología educativa hacia el año 2000. Revista Iberoamericana de educación, (5), 6796. https://rieoei.org/historico/oeivirt/rie05a03. pdf

Espín-Álvarez, E., y Freire-Muñoz, I. (2019). Relación entre el uso de internet para el entretenimiento y el aprendizaje escolar en estudiantes adolescentes del Ecuador. CienciAmérica, 8(1), 138-157. DOI:10.33210/ ca.v8i1.209

Espinel, E. (2020). La tecnología en el aprendizaje del estudiantado de la Facultad de Ciencias Químicas, Universidad Central del Ecuador. Revista Actualidades Investigativas en Educación, 20(2), 1-37. http://dx.doi. org/10.33210/ca.v8i1.209

Espinoza, C., Socorro, A., Soler, J., Hernández, H., y Guerra, C. (2020). Sistema estructurado de gestión del aprendizaje virtual de la Universidad Metropolitana del Ecuador. Revista Universidad y Sociedad, 12(5), 404-414. http://scielo.sld. $\mathrm{cu} /$ scielo.php? script=sci_arttextypid $=$ S2218$36202020000500404 \mathrm{ylng}=$ esynrm $=$ iso

Gallego, D., Cela, K., e Hinojosa, C. (2011). Una Mirada hacia el Ecuador frente a las tecnologías de la información y la comunicación en el ámbito educativo. Educación y futuro: Revista de investigación aplicada y experiencias educativas, 25, 115-132. http://hdl.handle.net/11162/94247
Gallo, G. (2020). La multimedia y el aporte al aprendizaje en los niños entre 6 a 10 años. Revista Formación docente, 3(1), 55-67. http:// revista.cae-internacional.com/index.php/rfd/ article/view/19/19

García, F. (2002). Software educativo: evolución y tendencias. Revista Aula, 14, 19-29. https://www. academia.edu/35192246/Software_educativo_ evoluci\%C3\%B3n_y_tendencias

García, V.; Pinargote, H. y García, E. (2019). Enseñanza virtual para niños con necesidades educativas especiales. Revista Encuentro Educacional, 26 (2), 173-191. https://www. academia.edu/download/67217328/34941_ Texto_del_articulo_59479_1_10_20210127.pdf

García-Peñalvo, F. y Seoane, A. (2015). Una revisión actualizada del concepto de eLearning. Décimo Aniversario. EKS, 16(1), 119-144. https://repositorio.grial.eu/bitstream/grial /390/1/1138-9737-2015-0016 -0001-0119-0144. pdf

Gil, D., Rosero, K. y Raza, C. (2011). Una Mirada hacia el Ecuador frente a las tecnologías de la información y la comunicación en el ámbito educativo. Educación y futuro: revista de investigación aplicada y experiencias educativas, (25), 115-132. https://dialnet.unirioja.es/ descarga/articulo/3775564.pdf

Gregorini, V. (2017). La cultura digital y las prácticas áulicas en la enseñanza de la Historia: renovaciones $y$ tensiones. XVI Jornadas Interescuelas/Departamentos de Historia. Departamento de Historia. Facultad Humanidades. Universidad Nacional de Mar del Plata, Mar del Plata, Argentina. https://cdsa. aacademica.org/000-019/698.pdf

Gros, B. (2000). Del software educativo a educar con software. Revista Quaderns Digital, 24, 440-482. http://www.quadernsdigitals.net/datos_web/ hemeroteca/r_1/nr_17/a_228/228.htm

Guanoluisa, D. y Herrera, L. (2018). Estudio de innovación tecnológica aplicada en procesos formativos con sistemas de gestión de aprendizaje (LMS) en la educación universitaria del Ecuador. Revista Ibérica de Sistemas e Tecnologias de Informação, (E15), 256-270. 
https://www.proquest.com/openview/e14 $91 \mathrm{dba} 22 \mathrm{~b} 2 \mathrm{c} 3 \mathrm{efc} 10564 \mathrm{~b} 5364 \mathrm{~b} 09 \mathrm{dd} / 1$ ?pqorigsite $=$ gscholarycbl $=1006393$

Hermann-Acosta, A., Apolo, D., y Molano-Camargo, M. (2019). Reflexiones y Perspectivas sobre los Usos de las Redes Sociales en Educación. Un Estudio de Caso en Quito-Ecuador. Información tecnológica, 30(1), 215-224. https://dx.doi. org/10.4067/S0718-07642019000100215

Hernández, Y. (2018). Desarrollo de soluciones para la informatización de la sociedad utilizando el framework de gestión de contenidos. Revista Universidad y Ciencia, 7(3), 175-188. http:// revistas.unica.cu/uciencia

Historia de la Informática. (02 de diciembre de 2010). Historia de las Redes Inalámbricas. https:// histinf.blogs.upv.es/2010/12/02/historia-de-lasredes-inalambricas/

Humanante-Ramos, P., Fernandez-Acevedo, J. y Jimenez, C. (2019). Aulas virtuales en contextos universitarios: percepciones de uso por parte de los estudiantes. Revista Espacios, 40 (2). http://ww.revistaespacios.com/a19v40n02/ a19v40n02p03.pdf

Lagos, G., Mora, K., Mejía, D., Peláez, R. y Peláez, J. (2019). M-learning, un camino hacia aprendizaje ubicuo en la educación superior del Ecuador. Revista Ibérica de Sistemas e Tecnologias de Informação, E18, 47-59. http://www.filosofia. ug.edu.ec/wp-content/uploads/docs/El\%20 m-learning\%20Gladis\%20Lagos\%20Reinoso. pdf

Lazo, C. y Gabelas, J. (2008). Ventanas de ocio: televisión y videojuegos. Chiasqui, 101, 50-55. http://hdl.handle.net/10469/13724

Leyva, M., Escobar, R., Espín, C., y Pérez, K. (2018). Facebook como herramienta para el aprendizaje colaborativo de la inteligencia artificial.Didasc@ lia: Didáctica y educación, 9(1), 27-36. http:// revistas.ult.edu.cu/index.php/didascalia/article/ view/728

López, J., Pozo, S., Morales, M., y López, E. (2019). Competencia digital de futuros docentes para efectuar un proceso de enseñanza y aprendizaje mediante realidad virtual. Edutec. Revista
Electrónica De Tecnología Educativa, (67), 1-15. https://doi.org/10.21556/edutec.2019.67.1327

López-Jiménez, D. (2010). Hábitos de consumo de Internet en Ecuador: Diferencias significativas entre estudiantes y no estudiantes. Comhumanitas, 2(1), 61-93. http://dspace.uhemisferios.edu.ec:8080/xmlui/ handle/123456789/362

Macías-Mendoza, F. (2015). Software educativo para potenciar la competencia comunicativa del inglés en la Universidad. Dominio de las Ciencias, 1(1), 85-95. DOI: http://dx.doi. org/10.23857/dc.v1i1.38

Magallanes, J., Rodríguez, Q., Carpio, Á., y López, M. (2021). Simulación y realidad virtual aplicada a la educación. RECIAMUC, 5(2), 101110. https://doi.org/10.26820/reciamuc/5.(2). abril.2021.101-110

Mantilla, D., Rodríguez, F., y Villacís, A. (2020). Diseño y desarrollo de un videojuego Educativo mediante una metodología ágil, como herramienta orientada a niños de 7 a 11 años para la prevención de la enfermedad de Chagas. Revista Ibérica de Sistemas e Tecnologias de Informação, (E33), 338350. https://www.proquest.com/openview/ 982cdec37fcce0ea94572d2450114410/1?pqorigsite $=$ gscholarycbl $=1006393$

Marcos, M. (1997). Aprender navegando, jugar a aprender. Educación y Biblioteca, 83, 13-18. https://gredos.usal.es/bitstream/ handle/10366/113421/EB09_N083_P13-18. pdf? sequence $=1$

Marizaca, V. (2015). Las tics en el proceso de enseñanza y aprendizaje de estudios sociales para mejorar la educación en el Ecuador (Tesis). UTMACH, Unidad Académica de Ciencias Sociales, Machala, Ecuador. http://repositorio. utmachala.edu.ec/handle/48000/3672

Marqués, P. (1996). El software educativo. En: J. Ferrés y P. Marqués. Comunicación educativa y Nuevas Tecnologías, 119-144. Praxis

Marqués, P. (1997). La informática en la enseñanza primaria. Revista Aula de innovación educativa, 67, 13-17. https:// 
scholar.google.com/citations?view_ op=view citationyhl=esyuser $=$ B 9vJ7gEA A A A Jycstart $=200$ ypagesize $=100$ ysortby= pubdateycitation for_ view $=$ B9vJ7gEAAAAJ:roLk4NBRz8UC

Martínez-Sanz, R., y Arribas-Urrutia, A. (2021). El rol de las redes sociales para futuros periodistas. Manejo, uso y comportamiento de estudiantes y profesores universitarios de Ecuador. Cuadernos Info, (49), 146-165. https://doi.org/10.7764/ cdi.49.27869

Matas, T. (2001). El multimedia. De la lectura a la interactividad. Revista Educación y Biblioteca, (124), 70-83. https://gredos.usal.es/bitstream/ handle/10366/118781/EB13_N124_P70-83. pdf? sequence $=1$

Mex, D., Hernández, L., Cab, J. y Castillo, M. (2021). El desarrollo cognoscitivo de la parábola según Bruner, con el empleo de software educativo. Revista Científica UISRAEL, 8(1), 137-155. https://doi.org/10.35290/rcui.v8n1.2021.402.

Mendoza-Zambrano, D., Tirado-Morueta, R. y Marín-Gutiérrez, I. (2017). Niveles de acceso a Internet de los estudiantes del bachillerato en Ecuador. Chasqui Revista Latinoamericana de Comunicación, (134), 391-410. https://www. redalyc.org/articulo.oa?id $=16057382023$

Montagud, N. (15 de abril de 2020). Investigación documental: tipos y características. Psicología y Mente. https://psicologiaymente.com/ miscelanea/investigacion-documental

Nivela, M., Otero, O., Espinosa, J., y Rodas, E. (2018). Diseño de software interactivo en las matemáticas. Revista Ciencia e Investigación, 3(1), 27-31. DOI: https://doi.org/10.26910/ issn.2528-8083vol3issCITT2017.2018pp27-31

Ocampo-Alvarado, J. y Ullauri-Ugarte, M. (2021). Factores para la aceptación y uso de un sistema de gestión del aprendizaje en estudiantes de una universidad ecuatoriana. Revista Electrónica Educare, 25(3), 1-21. https://www.revistas.una. ac.cr/index.php/EDUCARE/article/view/12733

Ocampo-Pazos, W., Garcia-Abad, J., MacasMacias, A., Carrasco-Ramirez, F., y CentenoLara, J. L. (2020). Videojuego Educativo para el pensamiento lógico-matemático en educación básica: Revisión sistemática de literatura. Revista Ibérica de Sistemas e Tecnologias de Informação, (E31), 513-531. https://www.proquest.com/ope nview/5345c115973c81b4d936519158a27511/1 ?pq-origsite $=$ gscholarycbl $=1006393$

Olguin, M., Rivera, I. y Hernández, E. (2006). Introducción a la Realidad Virtual. Polibits, (33), 11-15. https://www.redalyc.org/articulo. oa? $\mathrm{id}=402640446002$

Peláez-López, R., Morales-Roela, J., Lara-Vásconez, C., y Tutiben, M. (2018). Las tics y el uso de evea en instituciones de educación básica en Guayaquil-Ecuador. Revista Lasallista de investigación, 15(2), 131-140. https://doi. org/10.22507/rli.v15n2a10

Peñaherrera, M. (2011). Evaluación de un programa de fortalecimiento del aprendizaje basado en el uso de las TIC en el contexto ecuatoriano. RIEE. Revista Iberoamericana de Evaluación Educativa, 4(2), 72-91. http://www.rinace.net/ riee/numeros/vol4-num2/art4.pdf

Pérez, J. (1994). La teleenseñanza en la formación a distancia. Fundesco: Boletín de la Fundación para el Desarrollo de la Función Social de las comunicaciones, 150, 1-4. https://dialnet. unirioja.es/servlet/articulo? codigo $=5147638$

Ramírez, J. (2006). Las tecnologías de la información y de la comunicación en la educación en cuatro países latinoamericanos. Revista Mexicana de investigación educativa, 11(28), 61-90. http:// $\mathrm{www}$.scielo.org. $\mathrm{mx} / \mathrm{scielo}$.php? script $=\mathrm{sci}_{-}$ arttextypid=S1405-66662006000100061ylng=es ynrm=iso

Rogel, Y. y Verduga, A. (2013). Infraestructura Tecnológica de la Unidad Educativa Minerva de la Facultad de Filosofía, Letras y Ciencias de la Educación de la Universidad De Guayaquil. Estudio y Diseño para el Mejoramiento e Implementación de un Sistema Inteligente de Redes Wi-Fi para acceso a Internet en la Unidad Educativa Minerva (Bachelor's thesis). Universidad de Guayaquil, Guayaquil, Ecuador. http://repositorio.ug.edu.ec/handle/ redug/16797 
Ros-Martín, M. (2009). Evolución de los servicios de redes sociales en internet. El profesional de la información, 18 (5), 552-557. DOI: 10.3145/ epi.2009.sep. 10

Samaniego, J. (2016). Realidad Virtual en la Educación el Próximo Desafío. Journal of Science and Research: Revista Ciencia e Investigación, 1, 57-61. https://doi.org/10.26910/issn.25288083vol1issCITT2016.2016pp57-61

Sánchez, J. (2000). Aprender con Internet: Mitos y Realidades. Departamento de Ciencias de la Computación, Universidad de Chile. http://medios.educativos.umce.cl/externals/ documentos/Aprender_con_Internet.pdf.

Sangucho, D., y Baque, E. (2021). Educación ambiental basada en redes sociales: caso Universidad Tecnológica Israel. REVISTA ERUDITUS, 2(2), 9-28. https://doi. org/10.35290/re.v2n2.2021.450

Santo, J. y Chávez, M. (2020). Uso de los videojuegos en los adolescentes de las unidades educativas de la provincia de Manabí-Ecuador. Revista Atlante: Cuadernos de Educación y Desarrollo, (119). https://www.eumed.net/rev/atlante/2020/05/ videojuegos-adolescentes.html

Tapia-León, M., Peñaherrera-Larenas, F. y CedilloFajardo, M. (2015). Comparación de los LMS Moodle y CourseSites de Blackboard usando el modelo de aceptación tecnológica TAM. Revista Ciencia Unemi, 8 (16), 78-85. https://www. redalyc.org/articulo.oa? $\mathrm{id}=582663856010$

Tontaquimba, C. (2021). Desarrollo de un videojuego educativo en $2 \mathrm{D}$ para dispositivos móviles Android, enfocado al aprendizaje del ciberacoso en adolescentes entre los 12 a 14 años (Bachelor's thesis). Universidad Técnica del Norte, Ecuador. http://repositorio.utn.edu. ec/handle/123456789/11379

Tubay, J. y Oviedo, B. (2020). Las tecnologías inalámbricas y su impacto en el monitoreo de la actividad académica del talento humano en universidades. Centro Sur, 4(1). http://portal. amelica.org/ameli/jatsRepo/384/3841574017/ index.html

Urquiza, L., Auria, B., Daza, S., Carriel, F., y Navarrete, R. (2016). Uso de la realidad virtual, en la educación del futuro en centros educativos del Ecuador. Journal of Science and Research: Revista Ciencia E Investigación, 1(4), 26-30. https://doi.org/10.26910/issn.25288083vol1iss4.2016pp26-30

Valencia, J., Milla, A., y Machado, J. (1995). Propuesta de interconexión a Internet. Publicaciones Icesi, (54), 67-111. https://core.ac.uk/download/ pdf/229157191.pdf

Vidal, M., Gómez, F., y Ruiz, A. (2010). Software educativos. Educación Médica Superior, 24(1), 97-110. http://scielo.sld.cu/ scielo.php?script $=$ sci_arttextypid $=$ S0864 $21412010000100012 \mathrm{ylng}=\mathrm{esyt} \operatorname{lng}=\mathrm{es}$

Villalba, G., y Durán, P. (2010). Análisis e implementación del aprendizaje metódico, práctico de las tablas de multiplicar a través de la realidad virtual (Bachelor's thesis). Escuela superior Politécnica del Litoral, Guayaquil, Ecuador. http://www.dspace.espol.edu.ec/ xmlui/handle/123456789/13268 